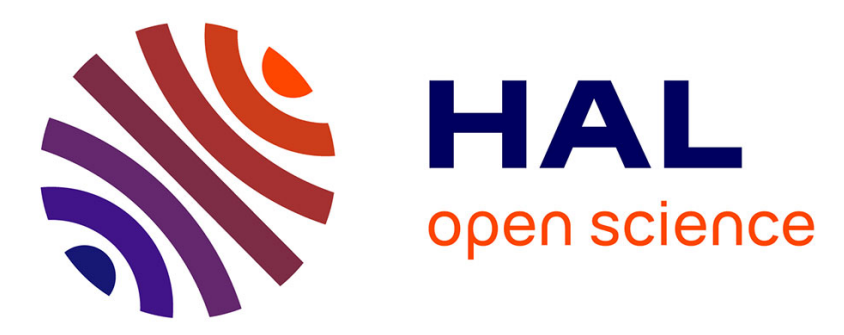

\title{
VALIDATION SUR MODĖLE RÉDUIT AU 1/100 DES CODES DE CALCUL DE PROPAGATION AU VOISINAGE D'UN SOL, AVEC ET SANS ÉCRAN, EN ACOUSTIQUE EXTÉRIEURE
}

Francois Barbier

\section{To cite this version:}

Francois Barbier. VALIDATION SUR MODÈLE RÉDUIT AU 1/100 DES CODES DE CALCUL DE PROPAGATION AU VOISINAGE D'UN SOL, AVEC ET SANS ÉCRAN, EN ACOUSTIQUE EXTÉRIEURE. Journal de Physique Colloques, 1990, 51 (C2), pp.C2-1161-C2-1164. 10.1051/jphyscol:19902272 . jpa-00230604

\section{HAL Id: jpa-00230604 https://hal.science/jpa-00230604}

Submitted on 1 Jan 1990

HAL is a multi-disciplinary open access archive for the deposit and dissemination of scientific research documents, whether they are published or not. The documents may come from teaching and research institutions in France or abroad, or from public or private research centers.
L'archive ouverte pluridisciplinaire HAL, est destinée au dépôt et à la diffusion de documents scientifiques de niveau recherche, publiés ou non, émanant des établissements d'enseignement et de recherche français ou étrangers, des laboratoires publics ou privés. 
COLLOQUE DE PHYSIQUE

Colloque C2, supplément au $n^{\circ} 2$, Tome 51, Février 1990

ler Congrès Français d'Acoustique 1990

VALIDATION SUR MODÈLE REDUIT AU 1/100 DES CODES DE CALCUL DE PROPAGATION AU VOISINAGE D'UN SOL, AVEC ET SANS ECRAN, EN ACOUSTIQUE EXTERIEURE

\author{
F. BARBIER* $(1)$ \\ Département Acoustique, Electricité de France, Direction des Etudes et \\ Recherches, 1 Avenue du Général de Gaulle, F-92141 Clamart Cedex, \\ France \\ "Conservatoire National des Arts et Métiers (CNAM), F-75141 Paris Ceđex \\ 03. France
}

\begin{abstract}
Résumé - Cette étude qui s'inscrit dans le cadre général de la propagation acoustique en milieu extérieur et de la protection de l'environnement est consacrée à la propagation sonore au-dessus des sols supposés plans, en présence ou non d'écrans plans dont les dimensions peuvent être finies. Les codes de calcul correspondants sont validés par modélisation physique sur maquette à l'échelle 1/100ème en milieu anéchoïque.

Abstract - These studies which concern the general context of outdoor sound propagation and environment protection have for subject the sound propagation above flat ground, with or without flat barriers, size of which can be finite or not. More, they confirm corresponding theoretical models of computation by means of physic modelisation on $1 / 100$ scale model in anechoïc room.
\end{abstract}

\title{
1 - INTRODUCTION
}

La modélisation de la propagation du bruit au voisinage d'une installation industrielle doit prendre en compte un nombre important de facteurs (réflexion sur le sol, réfraction par les couches de l'atmosphere, abstacles, turbulences, etc...). Ces contributions à la propagation font. en général, en raison de la complexité des équations mises en jeu, l'objet d'études séparées qui ne prennent pas en compte les différentes interactions possibles. Ces travaux /1/ ont eu pour but d'étudier les contributions a la propagation des sols et des écrans associés en confrontant des résultats expérimentaux obtenus par modelisation physique a des résultats théoriques délivrés par des codes de calcul exploités par EDF dans le cadre d'étude d'impact acoustique d'une installation industrielle.

Les modeles théoriques retenus font appel a une représentation exacte de la pression sonore émise par une source ponctuelle, au voisinage d'un sol plan et homogène; obtenue au moyen d'un terme d'onde de surface (Thomasson) /2/ ou à partir de potentiels de couches (Habault et Fillipi) 13\%. En présence d'un ecran plan de dimensions finies ou non, à l'effet de sol est associé l'effet d'écran calculé selon la théorie de la diffraction de Macdonald /4/. Pour chaque cas, le sol, supposé à réaction localisée, est caractérisé acoustiquement par son impédance selon un modèle à cinq paramètres /5/, analogue au modèle Delany-Bazley. Une méthode numerique /5,6/, basée sur la minimisation au sens des moindres carrés, permet l'identification des cinq parametres caractéristiques d'un sol, à partir de mesures de niveaux sonores en quelques points. Cette métrologie du sol, développée en vraie grandeur, a été adaptée à l'échelle 1/100ème pour les essaís sur modele réduit. Ce dernier, véritable maquette au 1/100ème en salle anéchoïque, permet de simuler, entre $20 \mathrm{~Hz}$ et $1 \mathrm{kHz}$, un sol réfléchissant et un sol partiellement absorbant, avec ou sans ecran. Une source sonore à plasma, modifiée pour les besoins expérimentaux, assure le rayonnement sphérique nécessaire à l'etude. Les erreurs systématiques induites par le processus de réduction d'échelle (absorption atmosphérique,...) sont compensées $/ 7 /$.

(1) Adresse actuelle : Département Recherche et Développement, Sociéte HPF, B.P.29, 74130 Bonneville France 


\section{2 - MODELES THEORIQUES}

Le système d'équations ci-dessous permet de poser le problème général de la propagation acoustique d'une onde harmonique au-dessus d'un sol plan d'impédance $Z$ finie, en atmosphère calme, isotrope et homogène :

$$
\left\{\begin{array}{l}
\left(\nabla^{2}+k^{2}\right) \Phi=-f, \quad z>0 \\
(\partial / \partial z+i \cdot k / z) \Phi=0, z=0 \\
\text { Condition de Sommerfeld }
\end{array}\right.
$$

Thomasson /2/ donne une représentation analytique du champ sonore, solution d'un tel système, au moyen de la somme de quatre termes :

$$
\Phi_{T}=\Phi_{D}+\Phi_{R}+\Phi_{G}+\Phi_{S}
$$

où $\Phi_{D}$ est le champ direct rayonné par la source en l'absence du plan frontière, $\Phi_{R}$ le champ réfléchi rayonné par la source image affectée du coefficient de réflexion d'onde plane, qui traduit l'atténuation et le déphasage subis par l'onde sonore lors de la réflexion sur l'interface des deux milieux air/sol. $\Phi_{G}$ représente une onde de sol, se propageant dans l'air au ras du sol. Ce terme décrit l'interaction entre le front d'onde sphérique et la surface plane. Il s'exprime à partir de la fonction d'erreur complémentaire et de termes qui intègrent la géométrie, la fréquence et la nature du sol. $\Phi_{\mathrm{s}}$ représente une onde de surface se propageant dans le sol, qui n'existe schématiquement que si les cotes de la source et du récepteur ne sont pas trop grandes.

Habault et Filippi /3/, par une approche différente, donnent une représentation analytique du champ sonore, egalement, à partir de la somme de quatre termes :

$$
\Phi_{T}=\Phi_{D}+B \cdot \Phi_{R}+\Phi_{S C}+\partial / \partial z\left(\Phi_{S C}\right)
$$

où $\Phi_{D}$ est le champ direct, $\Phi_{R}$ le champ réfléchi, et B une constante. $\Phi_{S C}$ est un potentiel de simple couche, ensemble de sources monopolaires portées par la surface plane parallèle au sol interceptant la source image. $/ / \partial_{z}\left(\Phi_{S C}\right)$ est un potentiel de double couche, dérivée par rapport a z d'un terme analogue au précédent, ensemble de sources dipolaires portées par la même surface.

Que l'on opte pour l'un ou l'autre de ces deux modeles de propagation, la réalisation des prévisions de l'effet de sol nécessite la connaissance des propriétés acoustiques des sols étudiés. Les modèles de sol les plus fréquemment envisagés sont les modèles a réaction localisée dont la connaissance passe par celle de leur impédance acoustique normale. Le modèle d'impédance retenu dans cette étude, à cinq paramètres, est le suivant :

$$
z=1+a_{1}(t / a 2)^{a 3}+i . a 4(f / a 2)^{a s}
$$

Si l'on pose al $=9,08 ;$ a3 $=-0,75 ;$ a4 $=11,9$ et as $=-0,73$ on retrouve le modèle d'impédance de DelanyBazley et le paramètre a2 est analogue à l'imperméabilité du sol considéré.

Dans le cas où l'on considère une propagation acoustique en présence d'un écran plan disposé sur le sol, il est nécessaire de prendre en compte non seulement les réflexions sur le sol, devant et derrière l'écran, mais encore les effets de diffraction sur le faite de l'écran et sur ses bords latéraux, lorsque ses dimensions sont finies. Ainsi, le champ sonore total s'écrit-il :

$$
\Phi_{T}=\Phi_{S R}+\Phi_{T R}+\Phi_{S X}+\Phi_{T X}+\Phi_{B O R D S}
$$

Le tableau 1, page suivante, donne l'ensemble des rayons sonores et des equations utilises pour calculer les quatre premières composantes du champ diffracté (ligne 2 à 5), selon (6). La ligne 1 explicite la valeur du champ, sans écran, qui sert de référence pour décrire l'effet d'écran. La colonne 1 montre les trajets sonores réels, avec leurs réflexions éventuelles, qui composent le champ total. La colonne 2, elle, indique les rayons équivalents utilisés pour le calcul des champs diffractés $\Psi_{i j}$. Ceux-ci, dans l'approximation du champ lointain, sont calcules a partir de la théorie de la diffraction de Macdonald /4/. Enfin. la colonne 3 du tableau 1 donne les équations des champs ainsi calculés, en tenant compte de l'influence du sol selon (3) et en négligeant onde de sol et onde de surface (approximation de Rudnick). Il est à noter que pour des raisons de simplification du graphisme, les effets de diffraction sur les bords latéraux de l'écran ne sont pas représentés dans le tableau 1, ci-après. En fait, ces effets sont pris en compte de manière semblable aux effets de diffraction sur le sommet de l'écran (4 premiers termes de (6)). Pour finir, notons que dans les expressions de ce tableau 1, les termes Pi sont les coefficients de reflexion d'onde plane, liés aux impédances acoustiques des sols situés de part et d'autre de l'écran. Ceuxci peuvent, d'ailleurs, être de nature différente. Les fanctions $F\left(w_{i}\right)$, fonctions de forme d'onde, ont, quant à elles, été évoquées précédemment (interaction front d'onde sphérique/surface plane). 
TABLEAU 1 : Couplage entre effet de sol et effet d'écran.

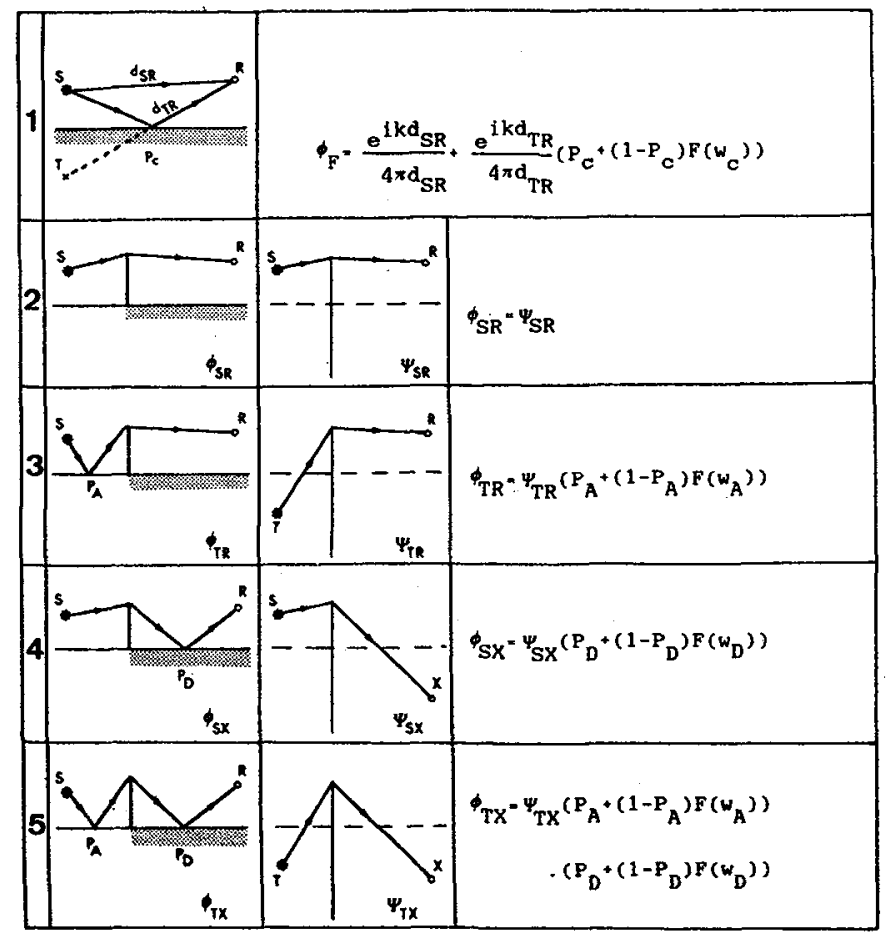

\section{3 - RESULTATS EXPERIMENTAUX}

L'ensemble des résultats expérimentaux de cette étude a eté obtenu sur le modele réduit PIGMEE (Programme d'Interprétation des Grandeurs acoustiques par Modélisation physique de l'Environnement Extérieur). Celuí-ci est constitue, essentiellement :

- d'un plateau plan d'environ $4 \mathrm{~m} \times 2 \mathrm{~m}$ placé en milieu anéchoïque,

- d'une source sonore à plasma emettant entre $2 \mathrm{kHz}$ et $100 \mathrm{kHz}$ pour permettre la simulation des effets entre $20 \mathrm{~Hz}$ et $1 \mathrm{kHz}$, en vraie grandeur,

- de microphones $1 / 4$ de pouce a condensateur dont la grille de protection est ôtée afin d'ameliorer la réponse en champ libre jusqu'à $100 \mathrm{kHz}$,

- d'un matériau absorbant, type feutre adhésif, simulant l'absorption d'un sol d'impédance finie,

- d'ecrans plans et minces parfaitement réfléchissants (tôle d'aluminium).

La figure 1(a), page suivante, indique, en termes de partie réelle et partie imaginaire, la valeur de l'impédance acoustique du feutre mesurée selon la méthode décrite dans $/ 5,6 /$. Après introduction des cinq parametres d'impédance, ainsi evalués, dans le code de calcul d'effet de sol, nous présentons un résultat de confrontation calcul-mesure à la figure 1(b). Les figures 2(a) et 2(b), quant a elles, présentent des confrontations théorie-expérience pour des propagations sonores au-dessus du même sol mais en présence d'un écran infiniment long ou, de dimensions finies, respectivement. Il est à noter que pour ces confrontations modélisation mathématique/modélisation physique, les écarts constatés en basse fréquence sont dûs aux variations de puissance acoustique de la source à plasma dans ce domaine.

\section{4 - CONCLUSION}

Les confrontations théorie-expérience réalisées dans le cadre de cette étude ont permis de verifier la pertinence des codes de calcul de propagation adoptés pour les sols plans, sans et avec ecran. Dans ce dernier cas, nous constatons que l'interaction entre la reflexion des ondes sonores sur le sol et la diffraction sur les bords d'un ecran est correctement simulee. Ainsi, l'aspect faisabilité d'une modélisation physique a une echelle aussi faible que 1/100ème a-t'il été validé, moyennant un certain nombre de précautions. Par exemple, nous avons pu constater la grande influence sur la qualité des confrontations calcul-mesure de la planéité du plateau simulant le sol sur le modèle réduit. Aussi un soin particulier a du être apporté dans sa réalisation. Nous avons en fait approché, là, les débuts d'une nouvelle étude : l'influence des 
reliefs sur la propagation sonore.

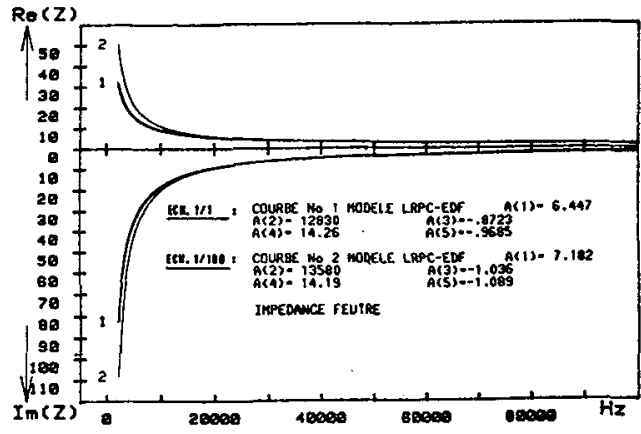

(a)

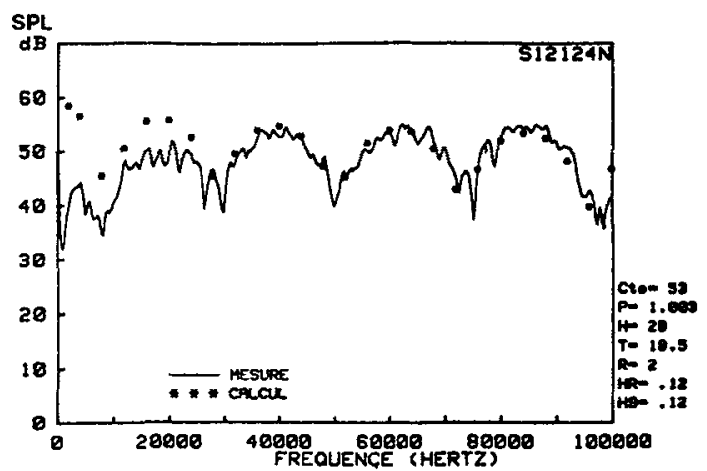

(b)

FIGURE 1 : (a) Evolution, en fonction de la fréquence, des parties réelle et imaginaire de l'impédance du sol partiellement absorbant du modele réduit, calculées a partir des 5 paramètres mesurés selon $/ 6 /$.

(b) Effet de sol: : confrontation calcul/mesure, pour un sol plan. d'impédance finie.

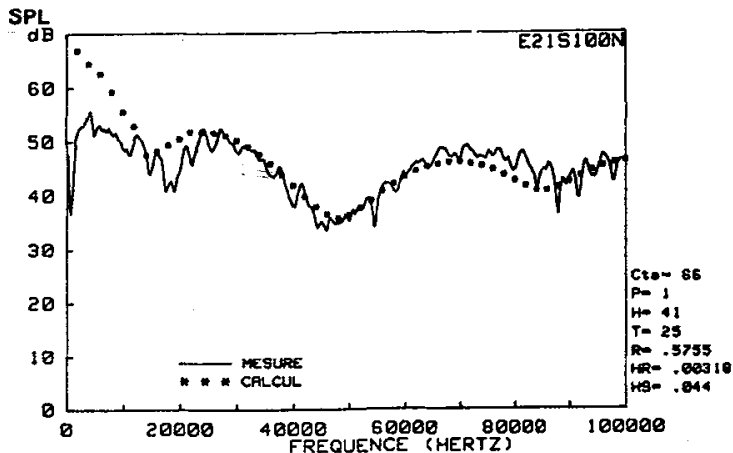

(a)

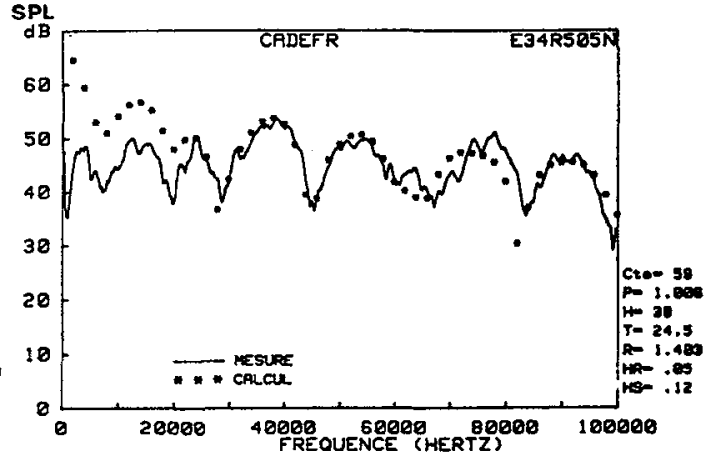

(b)

FIGURE 2 : Confrontations calcul/mesure, pour un sol plan d'impédance finie sur lequel repose

(a) un écran plan infiniment long,

(b) un écran plan de dimensions finies.

\section{REFERENCES}

/1/ Barbier F.- Validation sur modèle réduit au 1/100ène des codes de calcul de propagation au voisinage d'un sol, avec et sans ecran, en acoustique extérieure, Mémoire d'Ingénieur du Conservatoire National des Arts et Métiers, Paris, Mai 1989.

12/ Thomasson S.1.- Reflection of waves from a point source by an impedance boundary, J. Acoust. Soc. AM., 59(4), 1976, pp. 780-785.

13/ Habault D.- Etude de l'influence des sols sur la propagation sonore, Thèse de 3ème Cycle, Université de Provence, 1984.

14/ Isei T.- Absorptive noise barrier on finite impedance ground, 3. Acoust. Soc. Japan (E). 1(1), 1980, pp. 3-10.

/5/ Berengier M.- Propagation acoustique au voisinage du sol, Rapport du Laboratoire Central des Ponts et Chaussees, Paris, 1983.

/6/ Nonier E.- Méthode de mesure de l'impédance acoustique des sols, Rapport de la Direction des Etudes et Recherches d'Electricité de France, EDF-DER HE/22/4853, 1986.

/7/ Bass H.E., Sutherland L.C., Piercy J., Evans L.- Absorption of sound by the atmosphere, Physi cal Acoustics, Vol.XVII, 1984, pp. 145-232. 